\title{
Effect of Tuberculosis on Ovarian Reserve of Patients undergoing in vitro Fertilization
}

\author{
${ }^{1}$ Prabhjot Singh Hans, ${ }^{2}$ Mohan Lal Swarankar, ${ }^{3}$ Swati Garg, ${ }^{4}$ Manisha Chowdhary, ${ }^{5}$ Karnika Tiwari
}

\section{ABSTRACT}

Aim: To assess ovarian reserve, in infertile women with genital tuberculosis, planning to undergo in vitro fertilization (IVF) and to compare it with infertile women without genital tuberculosis, planning to undergo IVF.

Materials and methods: The study group consisted of 100 women with genital tuberculosis and the control group of 100 women who had no present or past history of tuberculosis. A diagnosis of genital tuberculosis was made based either on the results of tests performed from an endometrial aspiration sample or on histopathologic, hysterosalpingography, hysteroscopy, or laparoscopy findings. Basal ovarian reserve studies included measuring serum levels of follicle-stimulating hormone (FSH), luteinizing hormone (LH), and E2 on day 3 of a natural cycle. On the same day, the participants underwent a transvaginal ultrasound examination by means of a two-dimensional $5.0 \mathrm{MHz}$ probe fitted to a Toshiba Famio 5. Ovarian volume and number of antral follicles were estimated for each ovary on the same day of hormonal assessment. Control participants underwent the same tests on day 2 or 3 of their menstrual cycle. Unpaired or independent t-test and Chi-square test were used for statistical analysis.

Results: The present study highlights that women with genital tuberculosis have poor ovarian reserve in comparison to women of similar age without tuberculosis.

Conclusion: It can be concluded that there is no single absolute method of assessing ovarian reserve, but a combination of methods can closely predict the outcome of IVF cycles in women with genital tuberculosis.

Keywords: Genital tuberculosis, In vitro fertilization, Ovarian reserve.

How to cite this article: Hans PS, Swarankar ML, Garg S, Chowdhary M, Tiwari K. Effect of Tuberculosis on Ovarian Reserve of Patients undergoing IVF. Int J Infertil Fetal Med 2015;6(2):73-83.

Source of support: Nil

Conflict of interest: None

Date of received: $19-07-15$

Date of acceptance: 25-07-15

Date of publication: August 2015

\footnotetext{
${ }^{1,5}$ Postgraduate Student, ${ }^{2}$ Pofessor

${ }^{3}$ Professor and Head, ${ }^{4}$ Associate Professor

1-5Department of Obstetrics and Gynecology, Mahatma Gandhi Medical College and Hospital, Jaipur, Rajasthan, India
}

Corresponding Author: Prabhjot Singh Hans, Postgraduate Student, Department of Obstetrics and Gynecology, Mahatma Gandhi Medical College and Hospital, Sitapura, Jaipur Rajasthan, India, Phone: 01624-223787, e-mail: navsangeet. mangat566@gmail.com

\section{INTRODUCTION}

Little is known about the effect on ovarian reserve of infertile women harboring Mycobacterium tuberculosis. The infection heals by fibrosis, thus causing periovarian adhesions. These adhesions, in turn, may compromise ovarian reserve by impairing blood supply to the ovary.

Ovarian reserve is a term that is used to determine the capacity of the ovary to provide eggs that are capable of fertilization resulting in a healthy and successful pregnancy.

Ovarian reserve is defined as the quantity and quality of the follicles left in ovaries at any given time. ${ }^{2}$

While there is no known method for assessing the ovarian reserve of individual women, ${ }^{1}$ indirect determination of ovarian reserve is important in the treatment of infertility. ${ }^{2}$

Implication of ovarian reserve:

- To identify women who have sufficient ovarian reserve to make in vitro fertilization (IVF) using their own eggs

- To identify women who have little or no ovarian reserve

- To identify women who may be at risk of potentially OHSS (Yatess et al).

In IVF, the association of poor ovarian response due to diminished ovarian reserve with cycle cancellation and a significant decline in success rates is well known.,4

Accurate assessment of ovarian response potential before the patient enters an IVF program is, therefore, of pivotal importance. ${ }^{5}$ Apart from poor IVF outcome, patients with diminished ovarian reserve appear to be at greater risk for fetal aneuploidy compared to patients with normal ovarian reserve. ${ }^{6}$ Poor prognosis patients undergoing IVF have higher rates of chromosomal abnormal embryos as determined by preimplantation genetic diagnosis $(\mathrm{PGD})^{7,8}$ and exceedingly high rates of pregnancy loss, regardless of age. ${ }^{9}$

\section{Bologna Criteria for Reduced Ovarian Reserve}

At least two of the following three features must be present:

- Advanced maternal age ( $\geq 40$ years) or any other risk factor for poor ovarian response (POR) 
- A previous POR $(\leq 3$ oocytes with a conventional stimulation protocol)

- An abnormal ovarian reserve test (AFC 5-7 follicles or AMH 0.5-1.1 ng/ml) (Ferraretti et al).

\section{Ovarian Reserve Tests}

Over the past two decades, a number of ovarian reserve tests have been studied for their ability to predict outcome of IVF in terms of oocyte yield and occurrence of pregnancy. The accurate determination of ovarian reserve remains to be a challenge for reproductive physicians. ${ }^{1}$

\section{SCREENING OF OVARIAN RESERVE}

We review, here, all available tests of ovarian reserve by outlining their key strengths and weakness.

\section{Follicle Stimulating Hormone}

One of the first tests that were used to monitor ART cycles and offer an outcome prediction was the basal or early follicular phase follicle-stimulating hormone (FSH) level. Follicle-stimulating hormone is measured during cycle day 2 to 3, while the ovarian hormone levels are not yet elevated and a normal value is typically cited as lesser than $10 \mathrm{mIU} / \mathrm{ml}$.

The FSH level offers a reasonable understanding of the hypothalamus-pituitary-gonadal axis, as it responds to feedback from estradiol and progesterone.

\section{Advantages}

- This test is relatively cheap (400 INR) and only involves a blood draw.

- The results are usually available within 1 to 2 days.

- It has a very high specificity, upwards of $95 \%$

- At very high levels, FSH values are accurate predictors of poor response to ART treatment and failure of pregnancy, ${ }_{1}^{10}$ as they correlate with lower oocyte yield, higher rates of cancellation and lower rates of implantation and pregnancy. ${ }^{11}$

\section{Disadvantages}

- False negatives: Follicle-stimulating hormone values have to be interpreted in conjunction with an estradiol of lesser than 75 to $80 \mathrm{pg} / \mathrm{ml}$, to exclude a false lowering of FSH in response to an elevated estradiol, such as in cases of oral contraceptive use or pregnancy. ${ }^{12}$

- False positives: Although rare $(\sim 5 \%)$, cases of false positive FSH levels have been described. In the reported cases, patients with normal ovarian reserve had markedly elevated FSH values, as a result of either autoimmune or heterophile anti-FSH antibodies. Hence, clinical judgment is paramount in the interpretation of this test.
- Both the FSH and estradiol values are laboratory specific and practice-specific and should be interpreted individually in the setting of each patient's clinical scenario.

- Follicle-stimulating hormone has a pulsatile and circadian pattern of secretion that introduces the potential for measurement variability.

\section{Inhibin B}

Inhibin $B$ is one of the two subunits of inhibin, a $32 \mathrm{kDa}$ dimeric glycoprotein member of the transforming growth factor-beta (TGF- $\beta$ ), superfamily that is produced by the granulosa cells of preantral follicles and early antral follicles. Whereas inhibin B is the dominant subunit present during the early to-mind-follicular phase of the menstrual cycle, inhibin A levels rise during the latefollicular and mid-luteal phases. Inhibin acts primarily by binding and inhibiting, as its name implies, activin, and thus decreasing FSH production at the level of the anterior pituitary. As a result of this interaction, inhibin B has been developed as a marker of ovarian reserve.

\section{Advantages}

- This test is affordable and only involves a blood draw.

- The results are usually available within 1 to 2 days.

\section{Disadvantages}

- There is a lack of reliable assays. Only recently have second-generation assays been described, but they have not yet being incorporated into clinical practice.

- It had little value in predicting menopause, ovarian response and ART treatment outcomes. ${ }^{10}$

\section{Anti-Müllerian Hormone}

Similar to inhibin B, anti-Müllerian hormone (AMH) is another dimeric glycoprotein member of the TGF- $\beta$ superfamily and is produced by the granulosa cells of follicles that are up to $6 \mathrm{~mm}$ in diameter. Once follicles mature $\mathrm{AMH}$ is no longer secreted and this is reflected in its levels across life: AMH is barely detectable at birth, reached a peak after puberty and progressively decreases after that until it returns to undetectable levels in menopause. ${ }^{13}$ Anti-Müllerian hormone promotes FSH-dependent ovarian follicular growth and inhibits primordial follicle pool depletion. Serum levels greater than $0.7 \mathrm{ng} / \mathrm{mg}$ are considered normal, whereas values less than $0.3 \mathrm{ng} / \mathrm{ml}$ indicate DOR. Age-specific values are also available for $\mathrm{AMH}$.

\section{Advantages}

- This test is relatively cheap (1500 INR) and only involves a blood draw. 
- Anti-Müllerian hormone is the earliest and most sensitive biomarker of ovarian reserve decline with changes noted well before changes in FSH or inhibin B14:

- Unlike most other marker of ovarian reserve, AMH can be measured at anytime during the menstrual cycle as its value does not fluctuate. This allows for much higher scheduling flexibility and is not affected by cycle irregularities or difficulties with documentation.

- Anti-Müllerian hormone levels are independent of the hormonal status of the patient, i.e. pregnancy, use of oral contraceptives or gonadotrophin-releasing hormone agonist treatment. It can, thus, be measured and reliably interpreted during any of these states, without unnecessarily delaying diagnosis and initiation of treatment.

- Anti-Müllerian hormone age-specific values are potentially more informative than other markers were normal ranges are based on a cross-section of women independent of age.

- Anti-Müllerian hormone is a useful measure of ovarian reserve even in the context of elevated baseline FSH values and it allows for individualized ovulation induction protocols.

- Anti-Müllerian hormone is also reliable predictor of ovarian response to treatment, ovarian hyperstimulation syndrome as a result of ART and the onset of menopause. ${ }^{15-19}$

\section{Disadvantages}

- Anti-Müllerian hormone is run using ELISA assay, as opposed to an automated platform, therefore, requiring outsourcing for labs that may not run it daily. This, in turn, limits the test's widespread use because of a longer than 1 to 2 days waiting time to obtain results.

- Its value may be limited in certain populations, namely patients with obesity and polycystic ovary syndrome, as they are both characterized by ovulatory dysfunction. Whereas polycystic ovary syndrome patients tends to have higher $\mathrm{AMH}$ values, obese patients have lower AMH levels than their nonobese counterparts. $^{14,15}$

- Limited data exists on the predictive value of serum $\mathrm{AMH}$ in predicting pregnancy, although this is rapidly changing.

\section{Transvaginal Ultrasonography}

Another approach is to examine the ovaries by gynecologic ultrasound to determine their size as the ovaries depleted of follicles tend to be smaller ${ }^{15}$ and to evaluate the antral follicular count on day $2 / 3$ of menstrual cycle. ${ }^{16}$

\begin{tabular}{ll}
\hline Antral follicle count & Significance \\
\hline$<4$ & Poor reserve \\
$4-7$ & Low reserve \\
$8-12$ & Slightly reduced reserve \\
$>12$ & Normal \\
\hline
\end{tabular}

(Ghumman 2006. p. 135)

\section{AGE AND OVARIAN RESERVE}

The ovary is generally thought of as an egg bank from which the woman draws eggs during her reproductive life. The human ovary contains a population of primordial follicles. At 18 to 22 weeks post-conception, the female ovary contains its peak number of follicles (about 300,000 in the average case, but individual peak population's range from 35,000 to 2.5 million. ${ }^{20}$

\section{LIFE HISTORY OF OVARIAN FOLLICLE}

The size of the initial ovarian reserve is strongly influenced by genetics. Also, elevated androgen levels during prenatal development have an adverse effect on the early establishment of the ovarian reserve. ${ }^{21}$

Each menstrual cycle one egg cell is released by ovulation. In addition, the remaining follicles that were recruited towards maturation are lost by atresia. Few if any egg cells are replenished during the reproductive years. However, this loss by the menstrual cycle only accounts for approximately up to 10 egg cells per month, thus, accounting for only a small fraction of the actual loss of egg cells throughout the lifetime.

Variations in age at the occurrence of specific stages of ovarian aging. ${ }^{25}$

One additional contributory mechanism for the decline in the ovarian reserve with age appears to be a decreased gene expression of proteins involved in DNA repair by homologous recombination, such as BRCA1, MRE11, Rad51 and ATM. ${ }^{22}$

From studies on natural populations in which no consistent methods of birth control are applied, it has been shown that natural fertility starts to decline after the age of 30, accelerates in the mid-30s and will lead to sterility at a mean age of $41 .^{23-25}$

The reduction in female fertility can also be shown from contemporary population studies. The chance of not conceiving a first child within 1 year increases from under $5 \%$ in women in their early 20 s to approximately $30 \%$ or over in the age group of 35 years and older. So, although the majority of women of older age will obtain the desired pregnancy within a 1 year period, the chance of becoming subfertile increases $<6$ fold in comparison with very young women. ${ }^{26}$

Causes of decreased ovarian reserve:

- Genetic

- Age 
- Infection

- Pelvic

- Systemic (tuberculosis)

- Hormonal imbalance

- STD

- Gynecological surgeries

- Body weight

- Misce.

\section{Genital Tuberculosis (TB) is a Major Cause of Infertility in India, Accounting for 17 to $40 \%$ of Cases}

Tuberculosis, MTB, or TB (short for tubercle bacillus), in the past also called phthisis, phthisis pulmonalis, or consumption, is a common, and in many cases fatal, infectious disease caused by various strains of mycobacteria, usually M. tuberculosis. ${ }^{27}$

Tuberculosis typically attacks the lungs, but can also affect other parts of the body. It spreads through the air when people who have an active TB infection cough, sneeze, or otherwise transmit respiratory fluids through the air. ${ }^{28}$

Most infections do not have symptoms, known as latent tuberculosis. About one in 10 latent infections eventually progresses to active disease which, if left untreated, kills more than $50 \%$ of those so infected. Latency is a frequent outcome of untreated or incompletely treated M. tuberculosis infection, creating a longstanding reservoir of future disease and contagion. ${ }^{28}$

One third of the world's population is thought to have been infected with $M$. tuberculosis, ${ }^{29}$ with new infections occurring in about $1 \%$ of the population each year. ${ }^{26}$

Tuberculosis may infect any part of the body, but most commonly occurs in the lungs (known as pulmonary tuberculosis). Extrapulmonary TB occurs when tuberculosis develops outside of the lungs, although they may coexist with pulmonary TB as well. ${ }^{30,31}$

General signs and symptoms include: fever, chills, night sweats, loss of appetite, weight loss, and fatigue significant finger clubbing may also occur. ${ }^{32}$

\section{Pulmonary Tuberculosis}

If a tuberculosis infection does become active, it most commonly involves the lungs (in about $90 \%$ of cases). ${ }^{33,34}$ Symptoms may include chest pain and a prolonged cough producing sputum. About $25 \%$ of people may not have any symptoms (i.e. they remain 'asymptomatic'). ${ }^{33}$ Occasionally, people may cough up blood in small amounts, and in very rare cases, the infection may erode into the pulmonary artery, resulting in massive bleeding (Rasmussen's aneurysm). Tuberculosis may become a chronic illness and cause extensive scarring in the upper lobes of the lungs. The upper lung lobes are more frequently affected by tuberculosis than the lower ones. ${ }^{32}$ The reason for this difference is not entirely clear. ${ }^{1}$ It may be due either to better air flow, ${ }^{27}$ or to poor lymph drainage within the upper lungs. ${ }^{32}$

\section{Extrapulmonary}

In 15 to $20 \%$ of active cases, the infection spreads outside the lungs, causing other kinds of TB. ${ }^{35}$ These are collectively denoted as 'extrapulmonary tuberculosis.' Extrapulmonary TB occurs more commonly in immunosuppressed persons and young children. In those with HIV, this occurs in more than $50 \%$ of cases. ${ }^{36}$ Notable extrapulmonary infection sites include:

- The pleura (in tuberculous pleurisy),

- The central nervous system (in tuberculous meningitis),

- The lymphatic system (in scrofula of the neck),

- The genitourinary system (in urogenital tuberculosis), and

- The bones and joints (in Pott's disease of the spine), A potentially more serious, widespread form of TB is called 'disseminated' TB, commonly known as miliary tuberculosis. ${ }^{23}$ Miliary TB makes up about $10 \%$ of extrapulmonary cases. ${ }^{37}$

\section{DIAGNOSIS}

\section{Active Tuberculosis}

Diagnosing active tuberculosis based merely on signs and symptoms is difficult, as is diagnosing the disease in those who are immunosuppressed. A diagnosis of TB should, however, be considered in those with signs of lung disease or constitutional symptoms lasting longer than 2 weeks. A chest X-ray and multiple sputum cultures for acid-fast bacilli are typically part of the initial evaluation. ${ }^{34}$ Interferon- $\gamma$ release assays and tuberculin skin tests are of little use in the developing world. ${ }^{35}$ Interferon gamma release assay (IGRA) have similar limitations in those with HIV. ${ }^{38-40}$

A definitive diagnosis of TB is made by identifying M. tuberculosis in a clinical sample (e.g. sputum, pus, or a tissue biopsy). However, the difficult culture process for this slow-growing organism can take 2 to 6 weeks for blood or sputum culture. Thus, treatment is often begun before cultures are confirmed. ${ }^{41,42}$

Nucleic acid amplification tests and adenosine deaminase testing may allow rapid diagnosis of TB. ${ }^{38}$ These tests, however, are not routinely recommended, as they rarely alter how a person is treated. ${ }^{43}$ Blood tests to detect antibodies are not specific or sensitive, so they are not recommended. 


\section{Latent Tuberculosis}

The Mantoux tuberculin skin test is often used to screen people at high risk for TB. ${ }^{38}$ Those who have been previously immunized may have a false-positive test result. The test may be falsely negative in those with sarcoidosis, Hodgkin's lymphoma, malnutrition, or most notably, in those who truly do have active tuberculosis. ${ }^{27}$ Interferon gamma release assays (IGRAs), on a blood sample, are recommended in those who are positive to the Mantoux test. ${ }^{43}$ These are not affected by immunization or most environmental mycobacteria, so they generate fewer false-positive results. ${ }^{35}$ Interferon gamma release assays may increase sensitivity when used in addition to the skin test but may be less sensitive than the skin test when used alone.

\section{Tuberculosis and Ovarian Reserve}

Female genital tuberculosis is not uncommon in countries where pulmonary tuberculosis is widespread. In $92 \%$ of cases, genital tuberculosis is secondary to a focus in the lungs, lymph nodes, urinary tract, bones, or joints. Given the hormone-dependent nature of female genital tuberculosis, $90 \%$ of cases involve women under 40 years of age. The major presenting symptom is infertility $(45-55 \%)$,

Improved diagnostic techniques (e.g. endometrial curettage and biopsy, histologic examination of curettage, hysterosalpingography, bacteriologic examination of menstrual blood, laparoscopy, and ultrasonography) have resulted in increased awareness of genital tuberculosis. Another trend has been heightened awareness of the potential role of female genital tuberculosis in infertility.

Incidence of genital tuberculosis in infertile women is as high as $19 \%$.

Genital organs affected are:

- Fallopian tubes (about 90\%)

- Endometrium (50-60\%)

- Ovaries (10-30\%)

- Cervix, vagina and vulva (5\% for all combined). ${ }^{44}$

\section{MATERIALS AND METHODS}

The present cross-sectional study was conducted from December 1, 2012, to September 30, 2013, at the Outpatient Department of Obstetrics and Gynecology, Mahatma Gandhi Institute of Medical Sciences, Sitapura, Jaipur, India. The study group consisted of 100 women with genital tuberculosis and the control group of 100 women who had no present or past history of tuberculosis.

\section{Inclusion Criteria}

- All infertile patients who were planned for IVF. Participants were spontaneously menstruating with no signs or symptoms suggestive of menopause.

\section{Exclusion Criteria}

- Women younger than 22 years or older than 35 years BMI $<18.5$ or $>25$

- Women with polycystic ovaries

- Women with a single ovary

- A tubo-ovarian mass

- Ovarian endometrioma

- History of ovarian surgery.

The detailed medical history of each participant was taken, which included her menstrual and obstetric history, and a general medical examination was followed by an abdominal and vaginal evaluation.

A diagnosis of genital tuberculosis was made based either on the results of tests performed from an endometrial aspiration sample (the tests were a polymerase chain reaction to detect $M$. tuberculosis DNA, acid-fast bacilli staining, or liquid culture by means of mycobacteria growth indicator tubes and the Lowenstein-Jensen medium) or on histopathologic, hysterosalpingography, hysteroscopy, or laparoscopy findings. If any of the test for $\mathrm{TB}$ was found to be positive, the patient was considered TB positive.

Basal ovarian reserve studies included measuring serum levels of AMH on day 3 [Immunotech method (IOT) in $\mathrm{ng} / \mathrm{ml}$, FSH, luteinizing hormone ( $\mathrm{LH})$, and E2 [all these hormone levels in serum or plasma were tested using VIDAS automated quantitative test using enzyme-linked fluorescent assay (ELFA)] on day 3 of a natural cycle.

On the same day, the participants underwent a transvaginal ultrasound examination by means of a twodimensional $5.0 \mathrm{MHz}$ probe fitted to a Toshiba Famio 5 (Toshiba Medical Systems Ltd.). Ovarian volume and number of antral follicles were estimated for each ovary on the same day of hormonal assessment.

Control participants underwent the same tests on day 2 or 3 of their menstrual cycle.

Ethics clearance was obtained from the institute's Ethics Committee and written informed consent was provided by all participants prior to enrollment in the study.

\section{STATISTICAL ANALYSIS}

The software used for the statistical analysis were statistical package for social sciences (SPSS) version 21.0 and Epi-info version 3.0.

The statistical tests used were:

- Unpaired or independent $t$-test is used for comparison of mean value between 2 groups.

- Chi-square test is used to investigate whether distributions of categorical variables differ from one another. 
The $p$-value was taken significant when less than 0.05 ( $p<0.05)$ and confidence interval of $95 \%$ was taken.

\section{RESULTS}

The comparison of mean FSH, LH, Prolactin, TSH, E2 and $\mathrm{AMH}$ was done using unpaired t-test (Table 1). There was a significant difference $(p<0.05)$ in the mean E2 and $\mathrm{AMH}$ between TB negative and TB positive groups. The mean E2 was significantly $(p<0.05)$ more among TB positive group, whereas the mean $\mathrm{AMH}$ was significantly $(\mathrm{p}<0.05)$ more among TB negative group.

The comparison of mean no. of follicles counted was done using unpaired t-test (Table 2). There was a significant difference $(p<0.05)$ in the mean number of follicles counted between TB negative and TB positive groups. The mean number of follicles counted was significantly $(\mathrm{p}<0.05)$ more among TB negative group.
The comparison of mean ovarian length in $\mathrm{mm}$, ovarian width in $\mathrm{mm}$, oblique diameter and volume was done using unpaired t-test (Table 3).

There was a significant difference $(p<0.05)$ in the mean ovarian width (in $\mathrm{mm}$ ) between TB negative and TB positive groups. The mean ovarian width (in $\mathrm{mm}$ ) was significantly $(p<0.05)$ more among TB negative group.

There was a significant difference $(p<0.05)$ in the mean ovarian volume between TB negative and TB positive groups. The mean ovarian volume was significantly $(\mathrm{p}<0.05)$ more among TB negative group.

\section{DISCUSSION}

Studies investigating age-related changes in the hypothalamic-pituitary-ovarian axis have consistently demonstrated physiological and endocrine changes associated with reproductive aging. ${ }^{45}$ Ovarian reserve

PROFORMA

1. Patient

Regn. no.:

Date

2. Patient's name

3. Patient's age

A. $22-25$ yrs

B. $25-29$ yrs

C. $30-35$ yrs

4. Occupation of patient

5. Weight

6. Height

7. Husband's name

Age

Occupation

8. Duration of married life

9. Duration of infertility

10. Main cause of infertility
A. $<5$ yrs
B. 6-10 yrs
C. $11-15$ yrs
D. $16-20$ yrs

- Tubal factors

- Ovulatory disorder

- Endometrial factors

- Male factors

- Combined

- Unexplained

11. Type of infertility

- Primary

- Secondary

12. Chief complaints in brief

13. Menstrual history

- Duration of cycles

- Flow in days

- LMP

A. Menarche B. Present cycles:

- Regular/irregular

- Dysmenorrhea

- Others

14. Obstetric history

- Para

- Abortion/ectopic

- Last abortion

- Living

- Died: H/O abortion/premature delivery/IUD

- Last delivery

15. Past history
A. H/O tuberculosis (general/pelvic)
B. H/O STD
C. H/O DM, Htn, thyroid Ds
D. Past surgical history 
16. Family history

H/O DM, Htn, TB, obesity, infertility in sibling

17. Personal history:

- Smoking/tobacco chewing

- Alcohol intake: any other drugs

18. Previous management of infertility
A. Investigations
- Semen analysis
- HSG
- USG
- Hormonal profile: FSH/LH-Prolactin/TSH-E2-AMH
- Others.

B. Drugs

19. Diagnostic laparoscopy and/or hysteroscopy findings: cervical canal

$\begin{array}{llll}\text { Uterus } & \text { Fallopian tube } & \text { Ovaries } & \text { Others } \\ \text { Av } & \text { Normal } & \text { Normal } & \text { Tubo-peritoneal } \\ \text { Rv } & \text { U/L patent } & \text { Streak } & \text { Endometriosis } \\ \text { Normal size } & \text { B/L patent } & \text { Enlarged } & \text { Pelvic adhesions } \\ \text { Bulky } & \text { U/L hydrosalpinx } & \text { PCOD } & \text { Tuberculosis } \\ \text { Hypoplastic } & \text { B/L hydrosalpinx } & \text { Ovarian cyst } & \\ \text { Fibroid(s) } & \text { B/L ostia } & \text { Chocolate cyst } & \\ \text { Anomalies } & \text { TO mass } & \text { Follicuar cyst } & \\ \text { Capacity } & \text { U/L tubal block } & & \\ \text { Endometrium } & \text { B/L tubal block } & & \end{array}$

20. Basal hormonal levels

FSH

LH

Inhibin A

$\mathrm{AMH}$

21. USG

A. Number of follicles

Right ovary

Left ovary

B. Ovarian dimensions

Length

Width

Oblique diameter

22. Diagnosis of tuberculosis by

- TB PCR

- EB HPR

- TB IG M

- AFB culture

- TB feron gold test.

declines with age and is, therefore, an important determinant of the success of any fertility treatment. Ovarian reserve testing not only predicts fertility but also helps clinicians determine the best doses or protocols to help their patients achieve pregnancy. However, the available tests for ovarian reserve can predict oocyte quantity but not quality. These tests primarily focus on the ovarian response to gonadotropin stimulation. In countries like India, where tuberculosis is endemic, the conception rate is low (19.2\%), pregnancy outcome is poor, and the live birth rate is very low (7.2\%) among women with genital tuberculosis even after anti-tubercular therapy.

There is a paucity of articles focusing on the extent of ovarian damage in these women, but studies on assisted reproduction have shown poor gonadotropin treatment results, few oocytes retrieved, poor oocyte and embryo quality, and poor pregnancy outcome. ${ }^{39}$ Since genital TB often causes no physical symptom, asymptomatic infertile women should be investigated for silent or subclinical genital TB especially in high-risk populations. ${ }^{42}$ 
The present study assessed the reliability of several ovarian reserve tests when they are used in infertile women with tuberculosis who plan to undergo IVF, and compared the findings with those obtained for women with no present or past history of tuberculosis. By revealing significantly lower serum levels of biochemical markers of ovarian reserve, and a significantly lesser ovarian volume, in women with genital tuberculosis, the study also reveals a compromised ovarian reserve in these women.

On day 3 of the menstrual cycle the mean \pm SD values for FSH levels were significantly higher in the study than in the control group $(8.85 \pm 9.07 v s 11.35 \pm 7.36 \mathrm{IU} / \mathrm{L})$. Similarly, in the study done by Malhotra et $\mathrm{al}^{4}{ }^{43}$ the FSH levels were higher in TB positive patients (Table 4).

Of the various endocrine markers of ovarian reserve status, serum FSH level in the early follicular phase, with high basal levels of FSH suggesting a low ovarian reserve, is perhaps the most widely used. Women with low basal FSH levels respond favorably to ovarian stimulation, as a higher number of oocytes can be retrieved. Our study's results were similar to those published in a study by Gurgan et $\mathrm{al}^{46}$ and Malhotra et $\mathrm{al}^{43}$ in which women with genital tuberculosis had significantly higher FSH levels than control participants on day 3 of the menstrual cycle. In the present study, the mean FSH/LH ratio was significantly $(\mathrm{p}<0.05)$ more among TB positive group than TB negative group. Whereas the mean number of follicles counted was significantly $(p<0.05)$ more among TB negative group than TB positive group. Hence, increased levels of FSH can be correlated with decreased follicle count.

There was a significant difference $(p<0.05)$ in the mean $\mathrm{E} 2$ and $\mathrm{AMH}$ between TB negative and TB positive groups. The mean E2 was significantly $(p<0.05)$ more

Table 1: Mean FSH, LH, Prolactin, TSH, E2 and AMH in TB positive and TB negative patients

\begin{tabular}{|c|c|c|c|c|c|c|c|c|}
\hline \multirow[b]{2}{*}{ Hormones } & \multirow[b]{2}{*}{ Diagnosis } & \multirow[b]{2}{*}{ Mean } & \multirow[b]{2}{*}{$S D$} & \multirow[b]{2}{*}{$t$-value } & \multirow[b]{2}{*}{$p$-value } & \multirow[b]{2}{*}{ Mean difference } & \multicolumn{2}{|c|}{ 95\% Confidence interva } \\
\hline & & & & & & & Lower & Upper \\
\hline \multirow[t]{2}{*}{$\mathrm{FSH}$} & TB negative & 8.85 & 9.07 & -2.138 & $0.034^{*}$ & -2.496 & -4.798 & -0.193 \\
\hline & TB positive & 11.35 & 7.36 & & & & & \\
\hline \multirow[t]{2}{*}{$\mathrm{LH}$} & TB negative & 7.44 & 8.73 & 0.035 & 0.972 & 0.048 & -2.66 & 2.76 \\
\hline & TB positive & 7.39 & 10.61 & & & & & \\
\hline \multirow[t]{2}{*}{ Prolactin } & TB negative & 9.64 & 7.70 & -1.454 & 0.148 & -1.655 & -3.90 & 0.59 \\
\hline & TB positive & 11.29 & 8.39 & & & & & \\
\hline \multirow[t]{2}{*}{ TSH } & TB negative & 2.66 & 2.19 & 0.436 & 0.664 & 0.136 & -0.48 & 0.75 \\
\hline & TB positive & 2.52 & 2.22 & & & & & \\
\hline \multirow[t]{2}{*}{ E2 } & TB negative & 32.20 & 9.01 & -21.939 & $0.000^{* * *}$ & -31.563 & -34.40 & -28.73 \\
\hline & TB positive & 63.76 & 11.22 & & & & & \\
\hline \multirow[t]{2}{*}{$\mathrm{AMH}$} & TB negative & 3.54 & 2.06 & 4.824 & $0.000^{* * *}$ & 1.385 & 0.82 & 1.95 \\
\hline & TB positive & 2.15 & 2.00 & & & & & \\
\hline
\end{tabular}

Unpaired t-test; ${ }^{*}$ Significant difference, ${ }^{* * *}$ Very highly significant difference $(p \leq 0.001)$

Table 2: Mean number of follicles counted in TB positive and TB negative patients

\begin{tabular}{|c|c|c|c|c|c|c|c|}
\hline \multirow[b]{2}{*}{ Diagnosis } & \multirow{2}{*}{$\begin{array}{l}\text { No. of follicles } \\
\text { counted } \\
\text { Mean }\end{array}$} & \multicolumn{6}{|c|}{ 95\% Confidence interval } \\
\hline & & $S D$ & $t$-value & $p$-value & Mean difference & Lower & Upper \\
\hline TB negative & 11.10 & 1.09 & 8.547 & $0.000^{* * *}$ & 1.320 & 1.02 & 1.63 \\
\hline TB positive & 9.78 & 1.10 & & & & & \\
\hline
\end{tabular}

Unpaired t-test; ${ }^{* *}$ Very highly significant difference $(p \leq 0.001)$

Table 3: Mean and standard deviation of ovarian length, ovarian width, oblique diameter and volume in TB positive and TB negative patients

\begin{tabular}{|c|c|c|c|c|c|c|c|c|}
\hline & \multirow[b]{2}{*}{ Diagnosis } & \multirow[b]{2}{*}{ Mean } & \multirow[b]{2}{*}{$S D$} & \multirow[b]{2}{*}{$t$-value } & \multirow[b]{2}{*}{$p$-value } & \multirow[b]{2}{*}{ Mean difference } & \multicolumn{2}{|c|}{ 95\% Confidence interval } \\
\hline & & & & & & & Lower & Upper \\
\hline \multirow[t]{2}{*}{ Ovarian length $(\mathrm{mm})$} & TB negative & 26.17 & 3.62 & 1.077 & 0.283 & 0.678 & -0.56 & 1.92 \\
\hline & TB positive & 25.49 & 5.15 & & & & & \\
\hline \multirow[t]{2}{*}{ Ovarian width (mm) } & TB negative & 21.03 & 3.04 & 3.618 & $0.000^{\star * *}$ & 1.372 & 0.62 & 2.12 \\
\hline & TB positive & 19.65 & 2.26 & & & & & \\
\hline \multirow[t]{2}{*}{ Oblique diameter } & TB negative & 23.55 & 2.31 & 1.283 & 0.201 & 0.534 & -0.29 & 1.35 \\
\hline & TB positive & 23.02 & 3.46 & & & & & \\
\hline \multirow[t]{2}{*}{ Volume } & TB negative & 13244.53 & 1913.97 & 1.190 & $0.036^{*}$ & 1044.885 & -687.15 & 1776.92 \\
\hline & TB positive & 12199.64 & 3279.77 & & & & & \\
\hline
\end{tabular}

Unpaired t-test; *Significant difference $(p \leq 0.05) ;{ }^{* * *}$ Very highly significant difference $(p \leq 0.001)$ 
among TB positive group, whereas the mean $\mathrm{AMH}$ was significantly $(\mathrm{p}<0.05)$ more among TB negative group (Table 5).

The results of present study were in accordance with the study of Jirge et al, that concluded that mean AMH levels were low in TB positive group than TB negative group.

Beyond maternal age and smoking status, ovarian volume is a better measure of ovarian reserve than basal FSH. ${ }^{47}$ In their evaluation of ovarian diameter in the early follicular phase of the menstrual cycle, Bowen et $\mathrm{al}^{48}$ concluded that women with diminished ovarian reserve have significantly lower ovarian diameters than those with other factors for infertility. As age advances ovarian volume decreases. Women with small ovaries tend to respond poorly to ovulation induction and discontinue IVF-embryo transfer procedures at higher rates than those with ovaries of normal size for their age.

Women with small ovaries tend to respond poorly to ovulation induction and discontinue IVF-embryo transfer procedures at higher rates than those with ovaries of normal size for their age. Total ovarian volume and volume of the smallest ovary were found to be predictors distinct from maternal age for the smallest ovary, but not total ovarian volume, predicted clinical pregnancy occurrence $^{47}$ (Table 6). The mean ovarian volume was significantly (13244.53 \pm 1913.97 vs $12199.64 \pm 3279.77$ $\mathrm{mm}^{3} \mathrm{p} 0.036^{*}$ ) more among TB negative group than TB positive group Thus, TB negative group patients are expected to respond better to ovulation induction than TB positive group.

On cycle day 3 , the number of antral follicles provides prognostic information on the occurrence of ovarian response during hormone stimulation for IVF. ${ }^{5}$ In the present study, there was a significant difference $(\mathrm{p}<0.05)$ in the mean number of follicles counted between TB negative and TB positive groups. The mean number of follicles counted was significantly $(p<0.05)$ more among TB negative group.

The antral follicle count reflects ovarian reserve in women of reproductive age. It is accepted that a decrea- sing stock of primordial follicles results in a decrease in the number of antral follicles that will eventually provide an ovulatory follicle. ${ }^{43}$ During infertility treatment, this phenomenon manifests as a reduction in the number of dominant follicles developing in response to high doses of exogenous gonadotropins. In a prospective study, Bancsi et $\mathrm{al}^{49}$ found that antral follicle count was the best single predictor for poor ovarian response, better than patient age or basal FSH and inhibin B levels. A low count is a sign of ovarian aging that occurs earlier than the elevation of serum FSH levels. A meta-analysis has further shown antral follicle count to be a better predictor of poor ovarian response than basal FSH (Table 7). ${ }^{50}$

It could be argued that the best predictor of ovarian reserve for women with genital tuberculosis would be pregnancy. Endometrial damage is often present in these women, however, which could be a confounding factor. Another possibility would be to assess oocyte yield at IVF, but the different dosages and protocols used for ovarian stimulation could produce misleading results. This is why adopted model for ovarian reserve assessment was the matching of women with genital tuberculosis and control participants by age and body mass index.

Ovarian aging seems to have a major role in fertility outcome. It is related to a decrease in the quality of oocytes and a gradual decline in the number of primordial follicles. Patients with a diagnosis of genital tuberculosis should not be excluded from undergoing assisted reproduction procedures simply because of their disease. However, because test validation may facilitate both protocol decisions and prognosis, clinical and research endeavors need to focus on validating the tests used to assess ovarian reserve in women with genital tuberculosis who wish to attempt IVF. In conclusion, there is no single absolute method of assessing ovarian reserve, but a combination of methods can closely predict the outcome of IVF cycles in women with genital tuberculosis.

\section{CONCLUSION}

The present study highlights that women with genital tuberculosis have poor ovarian reserve in comparison

Table 4: Mean and standard deviation of FSH levels on day 3 of menstrual cycle

\begin{tabular}{|c|c|c|c|c|}
\hline Hormone & TB negative & TB positive & $p$-value & $\begin{array}{l}\text { 95\% Confidence interval for difference } \\
\text { between the two groups }\end{array}$ \\
\hline FSH (IU/L) & $8.85 \pm 9.07$ & $11.35 \pm 7.36$ & $0.034^{*}$ & -4.798 to -0.193 \\
\hline
\end{tabular}

*Significant different

Table 5: Mean E2 and AMH levels in TB positive and TB negative patients

\begin{tabular}{|c|c|c|c|c|}
\hline Dimension & TB negative & TB positive & $p$-value & $\begin{array}{l}95 \% \text { Confidence interval for difference } \\
\text { between the two groups }\end{array}$ \\
\hline E2 $(\mathrm{pg} / \mathrm{ml})$ & $32.20 \pm 9.01$ & $63.76 \pm 11.22$ & $0.000^{* * *}$ & -34.40 to -28.73 \\
\hline $\mathrm{AMH}(\mathrm{ng} / \mathrm{ml})$ & $3.54 \pm 2.06$ & $2.15 \pm 2.00$ & $0.000^{* * *}$ & $0.82-1.95$ \\
\hline
\end{tabular}

${ }^{* \star} p$-value $=0.000$ (very highly significant difference) 
Table 6: Mean ovarian volume in TB positive and TB negative patients

\begin{tabular}{lllll}
\hline Dimension & TB negative & TB positive & p-value & $\begin{array}{l}\text { 95\% Confidence interval for } \\
\text { difference between the two groups }\end{array}$ \\
\hline Volume & $13244.53 \pm 1913.97$ & $12199.64 \pm 3279.77$ & $0.036^{*}$ & -687.15 to 1776.92 \\
\hline
\end{tabular}

Table 7: Tuberculosis positive and TB negative patients based on age groups*

\begin{tabular}{lllll}
\hline & Diagnosis & Chi-square value & & \\
& TB negative & TB positive & & \multicolumn{2}{c}{-value } \\
\hline Age groups & Less than 25 years & $16(16.0 \%)$ & $37(37.0 \%)$ & 1.484 \\
& $25-29$ years & $39(39.0 \%)$ & $52(52.0 \%)$ & 0.476 \\
& $30-35$ years & $45(45.0 \%)$ & $100(100.0 \%)$ & \\
& Total & $100(100.0 \%)$ & $29.09 \pm 7.81$ \\
\hline
\end{tabular}

Chi-square test; *Significant difference $(p \leq 0.05)$

to women of similar age without tuberculosis. Since genital tuberculosis is a common cause of infertility in the Indian subcontinent, it is absolutely necessary to have knowledge of the effect of the disease on every organ of the reproductive system.

It can be concluded that there is no single absolute method of assessing ovarian reserve, but a combination of methods can closely predict the outcome of IVF cycles in women with genital tuberculosis.

\section{REFERENCES}

1. Broekemans FJ, et al. Ovarian reserve tests in infertility practice and normal fertile women. Maturitas 1998;30(2): 205-214.

2. Broekemans FJ, et al. A systematic review of tests predicting ovarian reserve and IVF outcome. Hum Reprod Update 2006;12(6):685-718.

3. Pellicer A, Lightman A, Diamond MP, Russell JB, DeCherney AH. Outcome of in vitro fertilization in women with low response to ovarian stimulation. Fertil Steril 1987; 47(5):812-815.

4. Jenkins JM, Davies DW, Devonport H, et al. Comparison of poor responders with good responders using a standard buserelin/human menopausal gonadotrophin regime for in vitro fertilization. Hum Reprod 1991;6(7):918-921.

5. Bancsi LF, Broekmans FJ, Eijkemans MJ, de Jong FH, Habbema JD, the Velde ER. Predictors of poor ovarian response in in vitro fertilization: a prospective study comparing basal markers of ovarian reserve. Fertil Steril 2002 Feb; 77(2):328-336.

6. Nasseri A, Mukherjee T, Grifo JA, Noyes N, Krey L, Copperman AB. Elevated day 3 follicle stimulating hormone and/or estradiol may predict fetal aneuploidy. Fertil Steril 1999;71(4):715-718.

7. Magli MC, Gianaroli L, Munne S, Ferraretti AP. Incidence of chromosomal abnormalities from a morphologically normal cohort of embryos in poor-prognosis patients. J Assist Reprod Genet 1998;15(5):297-301.

8. Munne S, Alikani M, Tomkin G, Grifo J, Cohen J. Embryo morphology, developmental rates, and maternal age are correlated with chromosome abnormalities. Fertil Steril 1995;64(2):382-391.
9. Levi AJ, Raynault MF, Bergh PA, Drews MR, Miller BT, Scott RT. Reproductive outcome in patients with diminished ovarian reserve. Fertil Steril 2001;76(4):666-669.

10. Broekmans FJ, Kwee J, Hendriks DJ, et al. A systematic review of tests predicting ovarian reserve and IVF outcome. Hum Reprod Update 2006;12(6):685-718.

11. Roberts JE, Spandorfer S, Fasouliotis SJ, et al. Taking a basal follicle-stimulating hormone history is essential before initiating in vitro fertilization. Fertil Steil 2005;83(1):37-41.

12. licciardi FL, Liu HC, Rosenwaks Z. Day 3 estradiol serum concentrations as prognosticators of ovarian stimulation response and pregnancy outcome in patients undergoing in vitro fertilization. Fertil Steril 1995;64(5):991-994.

13. Seifer DB, Baker VL, Leader B. Age-specific serum antimullerian hormone values for 17,120 women presenting to fertility centers within the United States. Fertil Steril 2011;95(2): 747-750.

14. Nelson SM, Anderson RA, Broekmans FJ, Raine-Fenning N, Fleming R, la marka A. Anti-Mullerin hormone: clairvoyance or crystal clesr? Hum Reprod 2012 Mar;27(3):631-636.

15. Broer SL, Dolleman M, Opmeer BC, et al. AMH and AFC as predictors of excessive response in controlled ovarian hyperstimulation: a meta-analysis. Hum Reprod Update 2011;17(1):46-54.

16. Broer SL, Dolleman M, Opmeer BC, et al. AMH and AFC as predictors of excessive response in controlled ovarian hyperstimulation: a meta-analysis. Hum Reprod Update 2011;17(1):46-54.

17. Broer SL, Eijkemans MJ, Scheffer GJ, et al. Anti-mullerian hormone predicts menopause: a long-term follow-up study in normo-ovulatory women. J Clin Andocrinol Metab 2011;96(8):253-259.

18. Pigny P, Merlen E, Robert $Y$, et al. Elevated serum level of anti-mullerian hormone in patients with polycystic ovary syndrome: relationship to the ovarian follicle excess and to the follicular arrest. J Clin Endocrinol Metab 2003;88(12): 5957-5962.

19. Buyuk E, Seifer DB, Illions E, et al. Elevated body mass index is associated with lower serum anti-Mullerian hormone levels in infertile women with diminished ovarian reserve but not with normal ovarian reserve. Fertil Steril 2011;95(7): 2364-2368.

20. Wallace WHB, Kelsey TW. Human ovarian reserve from conception to the menopause. PLoS ONE 2010;5(1):e8772. 
21. Richardson MC, Guo M, Fauser BCJM, Macklon NS. Environmental and developmental origins of ovarian reserve. Human Reprod Update 2013;20(3):353-369.

22. Titus S, Li F, Stobezki R, Akula K, Unsal E, Jeong K, Dickler M, Robson M, Moy F, Goswami S, et al. Impairment of BRCA1related DNA double-strand break repair leads to ovarian aging in mice and humans. Sci Transl Med 2013;5(172): 172-175.

23. van der Stege JG, van der Linden PJ (2001). Useful predictors of ovarian stimulation response in women undergoing in vitro fertilization. Gynecol Obstet Invest 2001;52:43-46.

24. Wood JW. Fecundity and natural fertility in humans. Oxf Rev Reprod Biol 1989;11:61-109.

25. Velde ER, Pearson PL. The variability of female reproductive aging. Hum Reprod Update 2002;8(2):141-154.

26. Abma JC, Chandra A, Mosher WD, Peterson LS, Piccinino LJ. Fertility, family planning, and women's health: new data from the 1995 National Survey of Family Growth. Vital Health Stat 1997;23(19):1-114.

27. Kumar V, Abbas AK, Fausto N, Mitchell RN. Robbins Basic Pathology. 8th ed. Saunders Elsevier; 2007. p. 516-522.

28. Konstantinos A. Testing for tuberculosis. Aust Prescriber 2010;33(1):12-18.

29. James E Gomez, John D McKinney M. Tuberculosis persistence, latency, and drug tolerance. Tuberculosis 2004;84(1-2): 29-44.

30. Tuberculosis fact sheet $\mathrm{N}^{\circ} 104$. World Health Organization. Nov 2010. Retrieved 26 July 2011. Available at: http://www. gramme-revit-sa.com/tuberculosis/what-is-tuberculosis.html.

31. Tuberculosis. World Health Organization 2002. Available at: http://www.who.int/mediacentre/factsheets/who104/en/ print.html.

32. Dolin, Mandell GL, Bennett JE, Raphael. Mandell, Douglas, and Bennett's principles and practice of infectious diseases 7th ed. Philadelphia, PA: Churchill Livingstone/Elsevier; 2010;pp. Chapter 250.

33. Lawn SD, Zumla AI. Tuberculosis. Lancet 2011 Jul 2;378(9785): 57-72.

34. Behera D. Textbook of pulmonary medicine. 2nd ed. New Delhi: Jaypee Brothers Medical Publishers; 2010. p. 457.

35. Jindal SK. Textbook of pulmonary and critical care medicine. New Delhi: Jaypee Brothers Medical Publishers; 2011. p. 549.

36. Kabra, Vimlesh Seth SK. Essentials of tuberculosis in children. 3rd ed. New Delhi: Jaypee Brothers Medical Publishers; 2006. p. 249.

37. Chen J, Zhang R, Wang J, Liu L, Zheng Y, Shen Y, Qi T, Lu H. Interferon-gamma release assays for the diagnosis of active tuberculosis in HIV-infected patients: a systematic review and meta-analysis. In Vermund, Sten H, editors Plos One 6, 2011.
38. Diseases, special programme for research and training in tropical. Diagnostics for tuberculosis: global demand and market potential. Geneva: world health organization on behalf of the special programme for research and training in tropical diseases 2006. p. 36.

39. Bento J, Silva AS, Rodrigues F, Duarte R. Diagnostic tools in tuberculosis. Acta Medica Portuguesa 2011 Jan-Feb;24(1): 145-154.

40. Tuberculosis. Clinical diagnosis and management of tuberculosis, and measures for its prevention and control. NICE Clinical guideline 117.

41. Amicosante M, Ciccozzi M, Markova R. Rational use of immunodiagnostic tools for tuberculosis infection: guidelines and cost effectiveness studies. The New Microbiologica 2010 Apr;33(2):93-107.

42. Namavar Jahromi B, Parsanezhada ME, Ghane-Shirazib R. Female genital tuberculosis and infertility. International Journal of Gynecology and Obstetrics Ž2001;75:269:27.

43. Malhotra N, Sharma V, Bahadur A, Sharma JB, Roy KK, Kumar S. The effect of tuberculosis on ovarian reserve among women undergoing IVF in India. Int J Gynecol Obstet 2012;117(1):40-44.

44. Malhotra BA, et al. Second-look hysteroscopy after anti tubercular treatment in infertile women with genital tuberculosis undergoing in vitro fertilization. Int J Gynecol Obstet 2010;108(2):128-131.

45. Hansen KR, Thyer AC, Sluss PM, Bremner WJ, Soules MR, Klein NA. Reproductive ageing and ovarian function: is the early follicular phase FSH rise necessary to maintain adequate secretory function in older ovulatory women? Hum Reprod 2005;20(1):89-95.

46. Gurgan T, Urman B, Yarali H. Results of in vitro fertilization and embryo transfer inn women with infertility due to genital tuberculosis. Fertil Steril 1996;65(2):367-370.

47. Syrop CH, Dawson JD, Husman KJ, Sparks AE, Van Voorhis BJ. Ovarian volume may predict assisted reproductive outcomes better than follicle stimulating hormone concentration on day 3. Hum Reprod 1999;14(7):1752-1756.

48. Bowen S, Norian J, Santoro N, Pal L. Simple tools for assessment of ovarian reserve: individual ovarian dimensions are reliable predictors of OR. Fertil Steril 2007;88(2):390-395.

49. Bancsi LF, Broekmans FJ, Eijkemans MJ, de Jong FH, Habbema JD, te Velde ER. Predictors of poor ovarian response in in vitro fertilization: a prospective study comparing basal markers of ovarian reserve. Fertil Steril 2002;77(2):328-336.

50. Hendriks DJ, Mol BW, Bancsi LF, Te Velde ER, Broekmans FJ. Antral follicle count in the prediction of poor ovarian response and pregnancy after in vitro fertilization: a metaanalysis and comparison. Fertil Steril 2005 Feb;83(2):291-301. 